\title{
Törnqvist and Roos reply:
}

The comment of Harada et al. [1] supports our claim expressed in our reply to Isgur and Speth [2] that crossed channel singularities are included in an average sense through the contact term and form factor $F(s)$, and that the $\sigma$ is needed to describe the data. There are in fact three recent analyses by different groups [3-5], not mentioned in Ref. [6] nor [7], which using different models find that a broad $\sigma$ with mass $\approx 500 \mathrm{MeV}$ is required by the data. These together with the present comment strengthen the actual existence of the light and broad $\sigma$.

In this connection a few general remarks are in order. First, we do not find it useful to study separately contributions from contact, crossed channel and resonances (such as $\sigma)$, since already by chiral symmetry [8] such Born terms are intimately intertwined with each others. Each diagram can separately violate the symmetry, and e.g. the contact and resonance term interfere destructively very strongly producing the Adler zeroes. Similar situations apply for gauge symmetries. Furthermore, after unitarization any such term in a sum of Born terms becomes connected to all other terms in the chain of higher order multiloop diagrams. The resulting amplitude is then (c.f. the N/D formalism) rather a product of $s$-channel resonances (in $D^{-1}(s)$ ) and crossed channel exchanges (in $N(s)$ ). In such a product a separation of a broad resonance pole from the "background" always involves analytic continuation, which is sensitive to fine details. Small changes in the amplitude at the real $s$-axis, where the data is, can lead to a very different picture in terms of separate terms in the sum.

Within a limited energy region (as from threshold to $1.5 \mathrm{GeV}$, which covers the region under discussion) there is very little freedom in chosing the $N(s)$ function, or its generalization in our multichannel framework: $\propto\left(s-s_{A i}\right) F^{2}(s)$ (See especially Sects 2.6-7 of Ref. [9]). Moreover, using flavor symmetry and data on $a_{0}(980)$ and $K_{0}^{*}(1430)$ this "freedom" is further reduced. In fact, since our simple parametrization of $F(s)$ fits the data, one can argue that $F(s)$ is a rough experimental representation of the crossed channel contributions. In accord with this, one observes that the parameter $k_{0}$ in $F^{2}(s)=\exp \left[-k^{2} / k_{0}^{2}\right]$ is of the right order of magnitude, expected from typical meson sizes. Using the formula of LeYaouanc et al. [10] (neglecting polynomial factors) this gives a radius of $R=\sqrt{6} / k_{0}=0.86 \mathrm{fm}$. Since our model does include crossed channel singularities in this approximate sense, we do not support the suggestion of Harada et al. [1], that our $\sigma$ parameters will change much in a more refined model. 
Of course, our conjecture that $\left(s-s_{A i}\right) F^{2}(s)$ is approximately of the correct form for the $N(s)$ functions should be proven by a more elaborate analysis, including sufficiently many of the crossed channel singularities, in addition to the already included chiral symmetry, flavor symmetry, unitarity and analyticity . Before the advent of such an analysis, we must be content with cruder models, such as that of Harada et al. [1,3], which supports, although does not prove, our conjecture.

[1] M. Harada, F. Sannino and J. Schechter, previous comment.

[2] N. Isgur and J. Speth, Phys. Rev. Lett. 772332 (1996) and N. A. Törnqvist and M. Roos, ibid 2333 (1996).

[3] M. Harada, F. Sannino and J. Schechter, Phys. Rev.D54 1991 (1996).

[4] S. Ishida et al., Prog. Theor. Phys. 95745 (1995), and KEK preprint 96-131 (Oct 1996), hep-th/9610359.

[5] R. Kamiński, L. Leśniak and J.-P. Maillet, Phys. Rev. D 503145 (1994) and R. Kamiński, L. Leśniak and K. Rybicki, Cracow preprint No. 1730/Ph, hep-ph 9606362, (to appear in Z. Phys. C).

[6] N.A. Törnqvist and M. Roos, Phys. Rev. Lett. 761575 (1996).

[7] R. M. Barnett et al., (the Particle Data Group), Phys. Rev. D54 1 (1996).

[8] De Alfaro et al., "Currents and Hadron Physics", North Holland (1973) p. 324-327.

[9] N.A. Törnqvist, Z. Phys. C 68647 (1995).

[10] LeYaouanc et al., Phys. Rev D8 2223 (1973).

Nils A. Törnqvist ${ }^{1)}$ and Matts Roos ${ }^{2)}$

1) Research Institute for High Energy Physics, SEFT, POB 9, FIN-00014, University of Helsinki, Finland

2) High Energy Physics Laboratory, POB 9, FIN-00014, University of Helsinki, Finland

Yours sincerely,

Nils Törnqvist, Matts Roos

Tornqvist@phcu.Helsinki.Fi 